Working

Paper

Department

of Economics

$\mathrm{Ca}^{\prime}$ Foscari University of

Venice

Sergio Currarini

Francesco Feri

Delegation Versus

Centralization: The Role

of Externalities 


\title{
Delegation Versus Centralization: The Role of Externalities
}

\author{
Sergio Currarini \\ University of Venice
}

Francesco Feri

University of Venice

\begin{abstract}
We study a simple contracting game with a principal and two agents. Contracts exert an externalities on non contractors. The principal can either contract both agents in a centralized manner, or delegate one agent to contract the other. We show that the choice of the principal depends on the sign of the externality. If this is positive, the principal prefers to delegate as long as the agency costs are not too high; if the externality is negative, the principal prefers to centralize for all sizes of agency costs.
\end{abstract}

\section{Keywords}

Contracts, Externalities, Centralization, Delegation.

\section{JEL Codes}

D02, D23, C71, C72

\author{
Address for correspondence: \\ Sergio Currarini \\ Department of Economics \\ Ca' Foscari University of Venice \\ Cannaregio 873, Fondamenta S.Giobbe \\ 30121 Venezia - Italy \\ Phone: (++39) 0412349133 \\ Fax: (++39) 0412349176 \\ e-mail: s.currarini@unive.it
}

This Working Paper (o QD se in inglese) is published under the auspices of the Department of Economics of the Ca' Foscari University of Venice. Opinions expressed berein are those of the authors and not those of the Department. The Working Paper series (o QD se in inglese) is designed to divulge preliminary or incomplete work, circulated to favour discussion and comments. Citation of this paper should consider its provisional character.

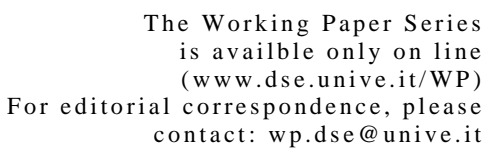

is availble only on line

contact:wp.dse@unive.it
Department of Economics
Ca' Foscari University of Venice
Cannaregio 873 , Fondamenta San Giobbe
30121 Venice Italy

Fax: ++390412349210 


\section{Introduction}

This paper studies the choice, made by a principal, between centralization and delegation of contracts with two agents. Each contract generates some value for the principal, who is therefore ready to make money transfers to agents in order to contract them. Two are the main ingredients of our analysis. First, only the principal is initially endowed with the necessary skills to make a contract effective. However, the principal can transfer these skills to the agents and allow them to sign contracts. Second, contracts exert an externality on non contractors, which can be positive (if the contract benefits the non contractor) or negative (if the contract hurts the non contractor).

We study a simple contracting game, in which the principal and the agents move only once, after which no further contacts between players can take place. We denote as "centralization of contracts" the choice, made by the principal, to directly contract both agents; we denote as "delegation of contracts" the choice, made by the principal, to sign up one agent and transfer her the contracting skills necessary to contract the second agent.

We show that the choice of the principal depends in a clear-cut way on the sign of the externality If this is positive, the principal strictly prefers to delegate one contract to an agent. This because, by doing so, the principal can decrease the outside option (and, therefore, the required payment) of one of the agent (the delegated), whose rejection would induce the complete absence of contracts. This ability of the principal to shape the agents' outside option depends on the assumed possibility of the principal to commit not to directly contract the second agent. This result survives for not too high agency costs of delegation. More precisely, the agency costs should not be larger than the externality generated by each contract.

If the externality is negative, results are less clear-cut in the absence of agency costs. Here, if agents accept or reject proposals sequentially, the principal is indifferent between delegation and centralization, and strictly prefers centralization for arbitrarily small agency costs to delegation.. This happens also if agents respond simultaneously and the principal can coordinate agents on his preferred Nash Equilibrium. In contrast, if agents can coordinate on their preferred Nash equilibrium, the principal strictly prefers centralization. Even in this case, however, the presence of arbitrarily small agency costs make the principal strictly prefer centralized contracts.

This paper is related to the recent literature on contracts with externalities. In particular, 
on the strategic manipulation of agents' outside options are based the papers by Segal (1999) and by Genicot and Ray (2006). Segal (1999) highlights the fact that the principal may sacrifice efficiency of the proposed contracts in order to ensure himself a more advantageous payoff distribution. In particular, the principal has an incentive to propose contracts that require actions from agents which are too small (under positive externalities) or too large (under negative externalities) with respect to the efficient levels. As in the present paper, this result depends on the assumption that the principal cannot write contingent contracts, that is, contracts whose effectiveness is made to depend on the acceptance of the contracts offered to other players.

In Genicot and Ray (2006), a principal chooses the timing of contracts in a setting of negative externalities. The focus there is on the preferred time profile of contracts for the principal. They show that the principal chooses not to offer all contracts in the first period, but rather to make acceptable offers in the first period to a subset of agents, in order to lower the outside option of the other agents, who can be contracted at better terms in following periods. Although there is no reference to the possibility of delegating contracts, the mechanism by which the principal shapes the agent outside option is similar to the one studied in our paper.

Finally, there exists a large literature on the advantages and disadvantages of delegation in the presence of agency costs (see, for instance, Mokkerje and Tsumagari (2004)). However, this literature mainly focuses on the solution of the trade off between the agency costs implied by delegation under asymmetric information and the advantages related to the use of better informed agents to perform a given task, or related to the relaxation of information processing constraints. Although the present paper does not investigate this trade-off, it contributes to this literature by pointing out, although in an extremely simplified framework, the possibility that the type of externalities exerted by contracts may affect the solution of the trade-off between delegation and centralization.

\section{The Contracting Game}

We consider a game with three players: a principal $(\mathrm{P})$ and two agents (A and $\mathrm{B})$. 


\subsection{Timing and Actions}

The principal moves first, and chooses whether to propose contracts to either A or B or both. The principal can also decide to contract one agent and to transfer to this agent the skills necessary to contract the second agent. If he chooses to do so, we say that he delegates to the chosen agent the task to contract the third agent. A contract consists of a real number $p$ expressing the payment required from (if $p<0$ ) or offered to (if $p>0$ ) the contracted agent. ${ }^{1}$

After $\mathrm{P}$ has moved, the contracted agents respond to the proposals made by $\mathrm{P}$. We here distinguish between two possible versions of the game, depending on whether agents A and B respond simultaneously or in sequence to a joint offer of $\mathrm{P}$. If agents respond in sequence we refer to the "sequential acceptance game", otherwise we refer to the "simultaneous acceptance game".

After P has moved, a subgame is identified by an element in the set

$$
\left\{\left(A, p_{A}\right),\left(B, p_{B}\right),\left(A^{d}, p_{A}\right),\left(B^{d}, p_{B}\right),\left(A, B, p_{A}, p_{B}\right)\right\}
$$

where the superscript $d$ indicates that the contracting skills have been transferred to the agent.

At the subgame $\left(A, p_{A}\right)$, agent $\mathrm{A}$ responds to $\mathrm{P}$ 's offer by either accepting or rejecting it, after which the game ends.

At the subgame $\left(B, p_{B}\right)$, agent $\mathrm{B}$ responds to P's offer by either accepting or rejecting it, after which the game ends.

At the subgame $\left(A, B, p_{A}, p_{B}\right)$, agent $\mathrm{A}$ responds to $\mathrm{P}$ 's offer by either accepting or rejecting it, after which agent $\mathrm{B}$ responds to $\mathrm{P}$ 's offer by either accepting or rejecting it.

At the subgame $\left(A^{d}, p_{A}\right)$, agent $\mathrm{A}$ either rejects P's offer, in which case the game ends, or accepts P's offer, in which case he chooses whether to propose a second contract $\left(B, p_{B}\right)$ to agent $\mathrm{B}$. In this second case, agent $\mathrm{B}$ observes the contract $\left(B, p_{B}\right)$ and decides wether to accept or reject the offer. In both cases the game ends.

At the subgame $\left(B^{d}, p_{B}\right)$ the same sequence of moves is possible, with the roles of $\mathrm{A}$ and B switched.

\footnotetext{
${ }^{1}$ We remark again that contingent contract, setting a payment which depends on the decision taken by the other agent, are ruled out. The possibility of such contract would invalidate all our results. One way to naturally rule out such contracts is to assume that agents cannot observe the contract proposed to other agents (see Segal (1999) for a discussion of this type of contracts and of the equilibrium notion to be used in this case).
} 
The game has a finite horizon, but is not finite because the range of payoff offers is not.

\subsection{Payoffs}

Each contract generates a positive value to the offerer. This assumption is without loss of generality since we are considering a game in which the offerer makes take it or leave it offers. Values are expressed by the function $v$, with $v(\varnothing)=0$ (no value is created without contracts). We denote by $v(P A \mid P B)$ the value generated by contract PA when also PB has been signed; similar expressions are obtained for other pairs of contracts. The function $v$ is assumed symmetric, in the sense that

$$
\begin{aligned}
v(P A) & =v(P B) ; \\
v(P A \mid P B) & =v(P B \mid P A) ; \\
v(A B \mid P A) & =v(B A \mid P B) ; \\
v(P A \mid A B) & =v(P B \mid B A) .
\end{aligned}
$$

Contracts generate value not only for the contractors, but also for the agent who is not involved in a contract. Such external effect may be positive or negative, according to the object of the contract. Consider, for instance, a case of political competition, in which a party $\mathrm{P}$ can either implement its preferred policy (a point on the real line), or contract other parties $\mathrm{A}$ and $\mathrm{B}$ to implement a mediated policy. Suppose the policies of $\mathrm{P}$ and $\mathrm{A}$ are both on one side of B's. If $\mathrm{P}$ and A sign such a contract, party B is hurt (negative externality) whenever A's policy is further away from B's policy than P's, and benefits (positive externality) whenever P's policy is further away.

We will denote by $\alpha$ the payoff accruing to agent A (resp., B) when agent B (resp., A) is contracted by P. Positive externalities correspond to the case $\alpha>0$, negative externalities correspond to the case $\alpha<0$.

Players' payoffs are finally given as follows as a function of signed contracts:

$$
\begin{aligned}
\left(P A, p_{A}\right) & \rightarrow u_{P}=v(P A)-p_{A} ; \quad u_{A}=p_{A} ; \quad u_{B}=\alpha . \\
\left(P B, p_{B}\right) & \rightarrow u_{P}=v(P B)-p_{B} ; \quad u_{A}=\alpha ; \quad u_{B}=p_{B} . \\
\left(P A, P B, p_{A}, p_{B}\right) & \rightarrow u_{P}=v(P A \mid P B)+v(P B \mid P A)-p_{A}-p_{B} ; \quad u_{A}=p_{A} ; \quad u_{B}=p_{B} . \\
\left(P A, A B, p_{A}, p_{B}\right) & \rightarrow u_{P}=v(P A \mid A B)-p_{A} ; \quad u_{A}=v(A B \mid P A)+p_{A}-p_{B} ; \quad u_{B}=p_{B} . \\
\left(P B, B A, p_{B}, p_{A}\right) & \rightarrow u_{P}=v(P B \mid B A)-p_{B} ; \quad u_{A}=p_{A} ; \quad u_{B}=v(B A \mid P B)+p_{B}-p_{A} .
\end{aligned}
$$




\section{Results}

We study the subgame perfect equilibria (SPE) of the contracting game in sections 3.1 and 3.2 for the cases of positive and negative spillovers. In section 3.3 we summarize the properties of these equilibria and draw some conclusions on the principal's choice between centralization and delegation of contracts.

\subsection{Positive Externalities}

\subsubsection{Game with Sequential Acceptance}

Without loss of generality (by symmetry of the payoff functions of the two agents), we assume that the principal always delegates to agent $\mathrm{A}$ in case he chooses to delegate. We proceed by backward induction.

Agent B. B faces a subgame in one of the following three classes:

a. $\left\{\left(A, B, p_{A}, p_{B}\right),(y e s)\right\}$ : both $\mathrm{A}$ and $\mathrm{B}$ have been offered a contract by $\mathrm{P}$, and $\mathrm{A}$ has accepted. In this case, $\mathrm{B}$ accepts if and only if $p_{B} \geq \alpha$.

b. $\left\{\left(A, B, p_{A}, p_{B}\right),(n o)\right\},\left\{\left(B, p_{B}\right)\right\}$ : both $\mathrm{A}$ and $\mathrm{B}$ have been offered a contract by $\mathrm{P}$, and $\mathrm{A}$ has declined, or $\mathrm{P}$ has proposed to $\mathrm{B}$ only. In these cases, $\mathrm{B}$ accepts if and only if $p_{B} \geq 0$.

c. $\left\{\left(A^{d}, p_{A}\right),\left(B, p_{B}\right)\right\}: \mathrm{P}$ has delegated $\mathrm{A}$ to contract $\mathrm{B}$, and $\mathrm{A}$ has accepted. In this case, $\mathrm{B}$ accepts if and only if $p_{B} \geq \alpha$.

Agent A. A faces a subgame in one of the following three classes:

a. $\left\{\left(A, B, p_{A}, p_{B}\right)\right\}$. If $\mathrm{A}$ accepts he gets $p_{A}$. If he does not accept, he gets $\alpha$ if $p_{B} \geq 0$ and 0 if $p_{B}<0$.

b. $\left\{\left(A, p_{A}\right)\right\}$. A accepts if and only if $p_{A} \geq 0$.

c. $\left\{\left(A^{d}, p_{A}\right)\right\}$. A can either reject and get 0 , or propose a contract to $\mathrm{B}$, who accepts if and only if $p_{B} \geq \alpha$. So, $\mathrm{A}$ gets from contract $\mathrm{AB}$ at most $v(A B \mid P A)-\alpha$. It follows that $\mathrm{A}$ accepts the delegation contract if and only if $p_{A} \geq \alpha-v(A B \mid P A)$. 
In figure 1 we depict the structure of payoffs as a function of agents choices after $\mathrm{P}$ has proposed centralized contracts $\left(A, B, p_{A}, p_{B}\right)$ (left tree) and delegated contracts $\left(A^{d}, p_{A}\right)$ (right tree).

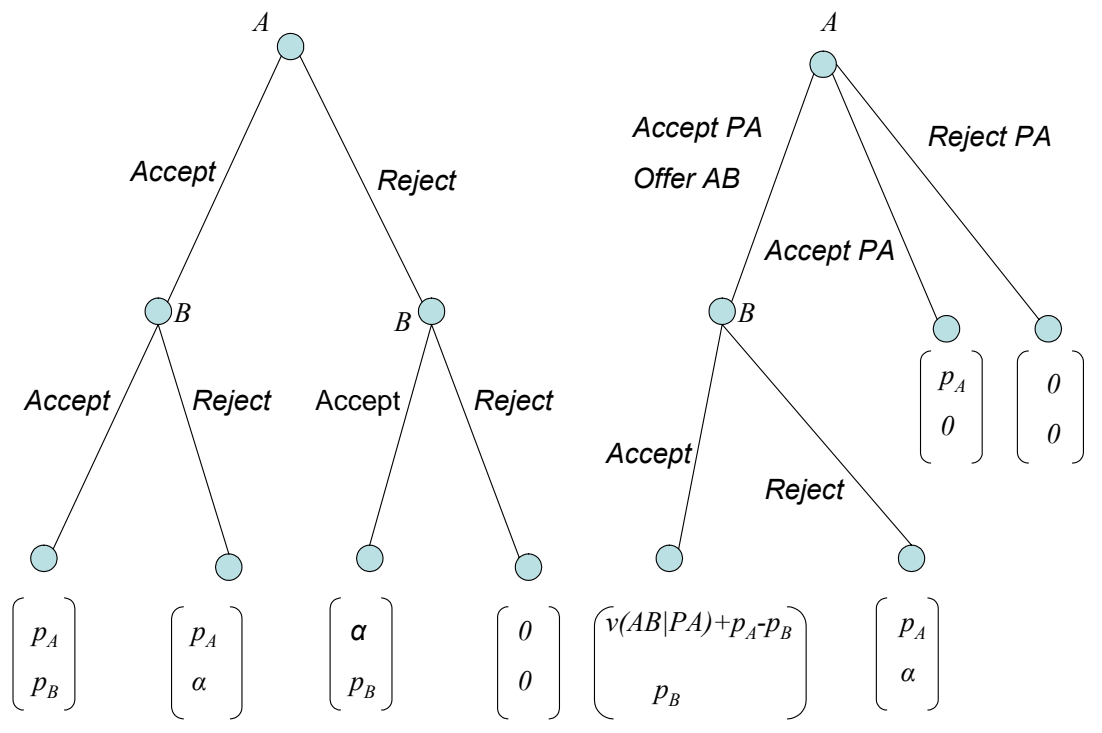

Figure 1: Histories and payoffs for centralized and delegated contracts.

The Principal: $\mathrm{P}$ can either make no proposal, or make proposal that either induce one contract, two "centralized" contracts $\mathrm{PA}$ and $\mathrm{PB}$, or the two contracts $\mathrm{PA}$ and $\mathrm{AB}$.

a. $\mathrm{P}$ can induce PA by offering $p_{A}=0$ and either not offering to $\mathrm{B}$ or offering $p_{B}<0$. Note that any offer $\alpha>p_{B} \geq 0$ would raise the outside option of $A$ to $\alpha$, and with it also the payment needed to make A accept the offer. By inducing PA the principal gets a payoff of $v(P A)$.

b. $\mathrm{P}$ can induce $\mathrm{PB}$ by offering $p_{B}=0$ and either not offering to $\mathrm{A}$ or by offering $\mathrm{A}$ $p_{A}<\alpha$. Either way, the principal gets a payoff of $v(P B)$.

c. To contract both agents, $\mathrm{P}$ needs to ensure that $B$ accepts the offer given that $\mathrm{A}$ has accepted. We have seen that this only happens when $p_{B} \geq \alpha$. Given this, we know from previous steps that $B$ will always accept P's offer. Therefore, A accepts if and only if $p_{A} \geq \alpha$. The maximal payoff for $P$ is therefore $v(P A \mid P B)+v(P B \mid P A)-2 \alpha$. 
d. If $\mathrm{P}$ offers a delegating contract to $\mathrm{A}$, we have seen that $\mathrm{A}$ accepts if and only if $p_{A} \geq \alpha-v(A B \mid P A)$. Therefore the principal gets a maximal payoff of $v(P A \mid A B)+$ $v(A B \mid P A)-\alpha$.

From the above analysis, it is clear that if there are no agency costs from delegation (that is, if $v(A B \mid P A)=v(P B \mid P A))$, then there can be two types of equilibria, depending on the shape of the function $v$.

If $v(P A)+\alpha>v(P A \mid A B)+v(A B \mid P A)$, then at all SPE there is one contract being signed. Note that this is the case in which have two contracts is socially inefficient.

If $v(P A)+\alpha<v(P A, A B)$, and therefore two contracts are socially efficient, the at all SPE the principal offers one contract with delegation, and the delegated agent contracts the other agent.

If $v(P A)+\alpha=v(P A, A B)$, then both $(\mathrm{PA})$ and $(\mathrm{PA}, \mathrm{AB})$ are equilibrium outcomes.

In particular, we note that the history in which the principal offers two contracts and both contracts are signed is never part of a SPE.

\subsubsection{Game with Simultaneous Acceptance}

When agents simultaneously respond to the principal's offers, the only difference from the above analysis is in the histories that go through the subgames $\left(A, B, p_{A}, p_{B}\right)$. Here we need to consider the Nash equilibria of the game in which agents simultaneously respond to $\left(p_{A}, p_{B}\right)$. These equilibria with acceptance are as follows (the first entry refers to A's action, the second to B's action): if $p_{A} \geq \alpha$ and $p_{B} \geq \alpha$ then $\{($ yes, yes $)\}$; if $p_{A} \leq 0$ and $p_{B} \leq 0$ then $\{(n o, n o),(y e s, n o)\}$. If $p_{A} \geq 0$ and $\alpha>p_{B}$, then $\{($ yes, no $\left.))\right\}$. If $p_{B} \geq 0$ and $\alpha>p_{A}$, then $\{(n o, y e s)\}$. Note that asymmetric offers by the principal are weakly dominated by the offer of a single contract. So, if $\mathrm{P}$ offers two contracts $\left(A, B, p_{A}, p_{B}\right)$, then these are both accepted if and only if $p_{A} \geq \alpha$ and $p_{B} \geq \alpha$, which leads to optimal offers of $p_{A}=p_{B}=\alpha$ by the principal. The SPE are therefore the same as in the sequential version.

\subsection{Negative Externalities}

Negative externalities are characterized by a negative value of $\alpha$. Again, and without loss of generality, we assume that the principal always delegates to agent $\mathrm{A}$ in case he chooses to delegate. 


\subsubsection{Sequential Acceptance}

Note that all the analysis of B'a and A's behaviour does not depend on the sign of $\alpha$, and therefore is valid here as well. We therefore only need to look at the principal's optimal choice to identify subgame perfect equilibrium outcomes.

The Principal: P can either make no proposal, or make proposal that either induce one contract, or two "centralized" contracts PA and PB, or the two contracts PA and AB.

a. $\mathrm{P}$ can induce $\mathrm{PA}$ by offering $p_{A}=0$ and either not offering any contract to $\mathrm{B}$ or offering $p_{B}<\alpha$. Note that any offer $p_{B} \geq 0$ would lower the outside option of $A$ to $\alpha$, but would also make $\mathrm{B}$ accept the contract. On the other hand, any offer $0>p_{B} \geq \alpha$ would not lower the outside option of A, but would make B accept the contract. So, by inducing PA the principal gets a payoff of $v(P A)$.

b. $\mathrm{P}$ can induce PB by offering $p_{B}=0$ and either not offering to $\mathrm{A}$ or by offering $p_{A}<\alpha$. Either way, the principal gets a payoff of $v(P B)$.

c. To contract both agents, $\mathrm{P}$ needs to ensure that $B$ accepts the offer given that $\mathrm{A}$ has accepted. We have seen that this only happens when $p_{B} \geq \alpha$. If $0>p_{B} \geq \alpha$, then $\mathrm{B}$ would reject if $\mathrm{A}$ rejects, which gives $\mathrm{A}$ an outside option of 0 . If $p_{B}=0, \mathrm{~B}$ accepts if A rejects, which gives $\mathrm{A}$ an outside option of $\alpha$. In both cases, The maximal payoff for $P$ is $v(P A \mid P B)+v(P B \mid P A)-\alpha$.

d. If $\mathrm{P}$ offers a delegating contract to $\mathrm{A}$, we have seen that $\mathrm{A}$ accepts if and only if $p_{A} \geq \alpha-v(A B \mid P A)$. Therefore the principal gets a maximal payoff of $v(P A \mid A B)+$ $v(A B \mid P A)-\alpha$.

From the above analysis, it is clear that if there are no agency costs from delegation (that is, if $v(A B \mid P A)=v(P B \mid P A)$ ), then there are two types of equilibria, one in which $\mathrm{P}$ offers contracts $(A, B, 0, \alpha)$ and one in which $\mathrm{P}$ offers $\left(A^{d}, \alpha-v(A B \mid P A)\right)$ and $\mathrm{A}$ offers $(B, \alpha)$. Proposing only one contract is never an equilibrium strategy for the principal as long as contracts produce non negative value.

\subsubsection{Simultaneous Acceptance}

When agents respond simultaneously to the principals offers, negative externalities generate multiple Nash Equilibria in the game played by agents. Suppose that $\mathrm{P}$ has proposed $p_{A}=0$ 
and $p_{B}=0$. Here, the profile $\{y e s, y e s\}$ is the unique equilibrium. However, if $p_{A}=\alpha$ and $p_{B}=\alpha$, then both $\{y e s, y e s\}$, and $\{n o, n o\}$ are Nash equilibria. If the principal could choose the equilibrium played by agents, he would opt for the first, while agents would like to coordinate on the second. Following Ray and Genicot (2006), we will say that in this second case the Nash Equilibrium is "coordination proof". Note also that, in this case, in order to obtain acceptance of both agents as the unique equilibrium the principal can propose $p_{A}=\varepsilon>0$ and $p_{B}=\alpha$ or $p_{A}=\alpha$ and $p_{B}=\varepsilon>0$.

We conclude that if we adopt the "coordination proofness refinement", then the principal obtains the maximal payoff of $v(P A, P B)-\alpha$ from delegating one agent to contract the other agent. If, in contrast, we allow the principal to choose the Nash Equilibrium played by agents, then $\mathrm{P}$ would get a maximal payoff of $v(P A, P B)-2 \alpha$ from offering two contracts and of $v(P A, P B)-\alpha$ from delegating. Offering two contracts would therefore survive as the unique SPE of the game.

\subsection{The Choice Between Delegation and Centralization}

The analysis of the previous section points to a clean-cut difference between the equilibrium structure of contracts when contracts exert positive or negative externalities on non contractors. In particular, whenever the second contract is productive enough and generates positive externalities, the principal strictly prefers to delegate this contract to an agent. If externalities are negative, the principal is indifferent between contracting both agents in a centralized manner and delegate the second contract to the agents. This indifference has been shown to disappear if the principal can induce agents to choose his most preferred Nash Equilibrium in the game with simultaneous acceptance.

There is, however, a more intuitive way of breaking this indifference in favor of a strict preference for centralization under negative externalities. Let us assume that delegation of contracts implies some kind of agency cost, that we label $C$ and is here defined as follows:

$$
C \equiv v(P B \mid P A)-v(A B \mid P A) .
$$

It is clear from the previous analysis that the presence of arbitrarily small agency costs would break the indifference of the principal in both games with simultaneous and sequential acceptance in favor of centralization, and would preserve the strict preference for delegation under positive spillovers.

The obtained results are summarized in the following two propositions. 
Proposition 1 If externalities are positive, the SPE of the contracting games with sequential and simultaneous acceptance coincide and induce the following structure of contracts:

i) if efficiency requires two contracts to be signed, and agency costs are smaller than the externality $(C<\alpha)$, then in the unique equilibrium the principal delegates one agent to contract the other agent, with the principal getting a payoff of $v(P A \mid A B)+v(A B \mid P A)-\alpha$, the delegated agent a payoff of 0 and the remaining agent a payoff of $\alpha$.

ii) if efficiency requires two contracts to be signed, and agency costs are larger than the externality $(C>\alpha)$, then in the unique equilibrium the principal offers two contracts and both agents accept, with the principal getting a payoff of $v(P A \mid P B)+v(P B \mid P A)-2 \alpha$, and both agents get a payoff of $\alpha$.

iii) if efficiency requires two contracts to be signed, and agency costs are equal to the externality $(C=\alpha)$, both the centralized and delegated contracts of point i) and ii) occur in equilibrium.

iv) if efficiency requires one contract, then in equilibrium only one contract is proposed and signed, with the principal getting a payoff of $v(P A)$ and the contracted agent a payoff of 0 and the remaining agent a payoff of $\alpha>0$.

Proposition 2 If externalities are negative and contracts generate positive value to the contractors:

i) For any amount of agency costs, that is whenever $C>0$, centralized contracts are the unique SPE outcome in both games with sequential and simultaneous acceptance.

ii) if the principal can coordinate agents on the preferred Nash Equilibrium then centralized contracts are the unique SPE outcome of the game with simultaneous acceptance.

iii) delegation is induced as the unique coordination proof SPE of the contracting games with simultaneous acceptance.

We conclude this section with two illustrative examples of the contracting situations analyzed in the paper, and of the results of propositions 1 and 2 at work.

Example 1 (Adjacent Gardens) Consider a street with two adjacent gardens, and assume that a well kept garden produces a utility of $\beta>0$ to the owner and a utility of $\alpha>0$ to the neighbor; a badly kept garden produces zero utility for both. We are assuming that the neighborhood externality is independent of the owner's decision to keep his own garden clean. 
Therefore the total utility deriving from only one nice garden in the street is $\beta+\alpha$ and that deriving from two nice gardens is $2(\beta+\alpha)$.

Finally, we assume that the only way to keep a clean garden is to pay a gardener. The gardener can supply garden services to both owners (the agents $A$ and B), either by proposing a contract separately to each owner (centralization), or by proposing a contract to owner A, under which $A$ has to convince his neighbor B to sign a contract to keep his own garden clean (delegation).

The optimal centralized contracts are such that the gardener proposes each owner to provide his services for a sum $\beta$. The payoff of the gardener is equal to $2 \beta$ and the owners receive $\alpha$ each. $A$ and $B$ have no incentives to deviate because their outside options are equal to $\alpha$. A larger payment than $\alpha$ is not an equilibrium, since each agent could profitably deviate.

In case of delegation, the gardener proposes $A$ to provide his services at a price $\beta+\alpha$ and transfers $A$ the skills to intermediate with $B ; A$ offers $B$ a price of $\beta$. The payoff of the gardener is equal to $2 \beta+\alpha, A$ receives 0 and $B$ receives $\alpha$. Both $A$ and $B$ have no incentive to deviate, since their outside option are 0 and $\alpha$, respectively.

It follows that the preferred choice for the gardener is the delegated contract structure, yielding a profit of $2 \beta+\alpha$, as opposed to the profit of $2 \beta$ obtained with centralized contracts.

Example 2 (Consumption Externalities) Consider two consumers, denoted by $A$ and $B$, consuming a good $X$. Each consumer is characterized by the demand function $p=1-x$. An amount $x$ of consumption by $A$ (resp, by $B$ ) generates a gross private utility of $x-\frac{x^{2}}{2}$ for $A$ (resp. for $B$ ) and a positive externality of $\delta x$ for $B$ (resp. for $A$ ). The externality from the other player's consumption disappears when one's own private consumption is positive (consumptions of the agents are substitute). Therefore if only one consumer consumes a quantity $x_{A}$ the total utility generated is equal to $x_{A}-\frac{x_{A}^{2}}{2}+\delta x_{A}$; if both consumers consume quantities $x_{A}$ and $x_{B}$ the total utility generated is equal to $x_{A}-\frac{x_{A}^{2}}{2}+x_{B}-\frac{x_{B}^{2}}{2}$; if no one consumes the total utility is zero.

There is monopolist that produces the good $X$ facing a constant marginal cost normalized to 0 . The monopolist has two possibilities to sell his production: the first is to propose a contract separately to the two consumers (centralization), the second one is to propose a contract to consumer $A$ and propose to him to contract with $B$ (delegation). A contract is a proposal to provide a given quantity $x_{I}$ for a payment $P_{I}$ that the monopolist receives from consumer $I \in\{A, B\}$. Given a contract between the monopolist and consumer $I$, the net 
private utility for $I$ is given by $x_{I}-\frac{x_{I}^{2}}{2}-P_{I}$, while the utility for the monopolist is given by $P_{I}$. In the following we assume that $\delta=\frac{1}{2}$.

The optimal centralized contracts for the monopolist solve the following problem:

$$
\begin{aligned}
& \max _{x_{A}, x_{B}, P_{A}, P_{B}} P_{A}+P_{B} \\
\text { s.t. } & \frac{1}{2} x_{B} \leq x_{A}-\frac{x_{A}^{2}}{2}-P_{A} \\
\frac{1}{2} x_{A} \leq & x_{B}-\frac{x_{B}^{2}}{2}-P_{B}
\end{aligned}
$$

The optimal solution for the monopolist is $x_{A}=\frac{1}{2}, x_{B}=\frac{1}{2}, P_{A}=\frac{1}{8}, P_{B}=\frac{1}{8}$. The monopolist's profit from these contract is $\frac{1}{4}$, while each consumer receives a net utility of $\frac{1}{4}$. Consumers $A$ and $B$ have no incentives to deviate, since their outside options are equal to the received utilities. By requiring sums which are strictly greater that $P_{A}$ and $P_{B}$ the monopolist would induce one consumer could to reject the contract (and not buy the good).

The optimal contracts with delegation for the monopolist solve the following problem:

$$
\begin{aligned}
& \max _{x_{A}, x_{B}, P_{A}, P_{B}} P_{A}+P_{B} \\
\text { s.t. } 0 \leq & x_{A}-\frac{x_{A}^{2}}{2}-P_{A} \\
\frac{1}{2} x_{A} \leq & x_{B}-\frac{x_{B}^{2}}{2}-P_{B}
\end{aligned}
$$

The optimal solution for the monopolist is $x_{A}=\frac{1}{2}, x_{B}=1, P_{A}=\frac{3}{8}, P_{B}=\frac{1}{4}$. The monopolist's profit from these contracts is $\frac{5}{8}$, consumer $A$ 's net utility is zero, while consumer $B$ 's net utility is $\frac{1}{4}$. Consumers $A$ and $B$ have not incentives to deviate, since they receive utilities equal to their outside options. By requiring sums which are strictly greater that $P_{A}$ and $P_{B}$ the monopolist would induce one consumer could to reject the contract (and not buy the good).

\section{Concluding Remarks}

We have studied a simple example of contracting situation, in which the principal wishes to contract two agents to perform a given task that he cannot perform by himself. The main feature of our framework is that contracts generate externalities on non contractors, who can be hurt by or benefit from the contract signed by the other agent. 
The simple one principal-two agent game we considered has been useful in providing an intriguing intuition on the effect of externalities on the equilibrium structure of contracts. We have seen how a positive externality makes the principal prefer a structure of delegated contracts, which lowers the outside option of the "middle" agent, whose refusal to sign the contract would lead, differently from the case of a centralized structure, to the failure to perform the task. If the externality is negative, the principal has a strict preference for centralization only when agents respond simultaneously and can be coordinated on the preferred Nash equilibrium for the principal; otherwise the principal is indifferent. Strict preference for centralization reappears with the presence of (even arbitrarily small) agency costs.

One interesting question is whether the game studied in this paper captures, despite its extremely simple structure (finite horizon, commitment possibilities, absence of contingent contracts), some aspects of the behaviour of agents in a contractual situation with externalities. The answer is, of course, object of experimental work, which we plan to do as the next step of this research.

\section{References}

[1] Genicot, G. and D. Ray (2006), "Contracts and Externalities: How Things Fall Apart", Journal of Economic Theory, forthcoming.

[2] Mookherjee, D. and M. Tsumagari (2004), "The Organization of Supplier Networks: Effects of Delegation and Intermediation", Econometrica 72(4): 1179-1219

[3] I. Segal (1999), "Contracting with externalities, Quart. J. Econ. 114: 337-388. 